\title{
Application of a Multi-Agent System for Risk Assessment with Fuzzy Background Information
}

\author{
Muhamediyeva D.T., Urokov Sh.
}

\begin{abstract}
The article offers the results of a study of various multiagent systems on the example of a number of models and multiagent implementations for risk assessment with fuzzy initial information. General construction methods and issues are related to their behavior, criteria for the quality of system performance that highlighted. The regularities, interrelations between the properties and parameters used when specifying a multi-agent intelligent system are defined. Developed approaches for processing complexly structured information. Algorithms for constructing a multi-agent intelligent risk assessment system have been developed.

Keywords. Artificial intelligence, hybrid intellectual systems, neuro-fuzzy models, evolutionary algorithms, multi-agent intelligent system
\end{abstract}

\section{INTRODUCTION}

For complex processes are characterized by uncertainty (inaccuracy, non-stochasticity, incompleteness, vagueness) in the initial information and situations of the external and internal environment, it is usually not possible to build simple adequate mathematical models. Information about the parameters of such processes is expressed by experts in the form of words and sentences, i.e. in linguistic form. In such cases, it is advisable to use systems of modeling, decision making and management using the tools of soft computing technology (Soft Computing) [1,2].

As the complexity of the system increases, there arises a difficulty in determining the correct set of rules and membership functions to describe adequately the behavior of the system. Fuzzy systems suffer from the drawbacks of extracting additional knowledge from the results of the experiment and adjusting fuzzy rules to improve the quality of the system. When evaluating alternative decision making options for risk assessment under uncertainty, the problem arises of developing fuzzy models based on fuzzy inference rules.
The advantage of fuzzy logic is the ability to use expert's knowledge about this object in the form of "if" inputs ", then" outputs "". In the process of developing a fuzzy risk assessment model is based on the findings of fuzzy rules, researchers often face the problem of finding approximate solutions to ill-posed problems. It should be noted that the methods intend to solve incorrect problems of decision-making support systems have been developed only for a number of special cases of models (for example, for models based on classical logic). At the same time, there is no general approach to solve fuzzy logic problems for arbitrary fuzzy systems.

Therefore, the analysis of risk assessment tasks in fuzzy conditions, as well as modeling and algorithmic support for solving ill-posed problems, formalized in the process of this analysis, are relevant for modern decision support systems.

Decision making problems in assessing the risk of natural, man-made and environmental disasters are discussed in the works of V.I. Norkin and Yu.M.Ermolyev [3], V.S. Mikhalevich and P.S. Knopov, I.V. Sergienko and V.M. Yanenko [4]. Formation of risk in economic terms is considered in the works of A.O. Nedosekin [5]. Management issues that related to risk prevention measures are discussed in the work of Yu.M. Ermolyeva [3]

The analysis of existing methods for solving problems of risk assessment has shown that they are based on the insufficiency of computational capabilities and the lack of necessary information about the conditions of the problem. Therefore, in such cases it is advisable to use fuzzy mathematical methods.

2. Statement of the problem. Let a training sample of fuzzy experimental data that given. $\left(X_{r}, y_{r}\right), \quad r=\overline{1, M}, \quad$ where $X_{r}=\left(x_{r, 1}, x_{r, 2}, \ldots, x_{r, n}\right)$ - fixed values of the input n-dimensional vector and $y_{r^{-}}$the corresponding values of the output vector.

Based on fuzzy inference rules, you need to build a model based on fuzzy inference rules:

Revised Manuscript Received on July 25, 2019.

Muhamediyeva D.T., Urokov Sh.

Tashkent university of information technologiesTashkent, Uzbekistan 
(1)

$$
\mathrm{Y}_{p=1}^{k_{j}}\left(\sum_{i=1}^{n} x_{i}=a_{i, j p}-\text { with weight }\right) \rightarrow y_{j}=b_{m_{0}}+b_{m_{1}} x_{1}^{j}+\ldots+b_{m_{n}} x_{i}^{j}
$$

In the process of building a model, you need to find such values of the coefficients of the rules $B=\left(b_{i j}\right), i=\overline{1, n}, j=\overline{1, m}$, at which the minimum of the following expression is reached: $\sum_{r=1, M}\left(y_{r}-y_{r}^{f}\right) \rightarrow \min$, where - $y_{r}^{f}-\left(X_{r}\right)$ is the result of a fuzzy output in the row of the selection with the parameter B.

We rewrite problem (2) in the following matrix form: find a vector B such that the condition

$$
E=\left(Y-Y^{f}\right)^{T} \cdot\left(Y-Y^{f}\right) \rightarrow \min
$$

The solution of the problem (3) corresponds to the solution of the following equation:

$$
Y=A \cdot B
$$

\section{MULTI-AGENT SYSTEMS}

Recently, multi-agent systems (MAS) are one of the most important and promising areas for the development of information and communication technologies. This is due to the ever-increasing complexity, spatial distribution of modern information systems, organizations, objects under study [6].

In parallel with the practical implementation of a theoretical analysis of the principles of multi-agent systems. Since the MAS are used in many different areas, there are different approaches to the study of MAS depending on the issues facing the researchers. The logical approach to the description and research of MAS is very popular, which consists in describing their work with the help of various logical structures, introducing special operators to describe the work of agents. A probabilistic approach is also used, in which some elementary steps of the system's operation are considered to be exposed to random factors (for example, a delay in the transfer of information, or individual decisions of agents) [7].

However, many models built using the agent-based approach are purely imitative, and the algorithms (if we are talking about AI systems) are purely heuristic. The task of theoretical substantiation of their effectiveness and evaluation of their behavior remains unsolved. All this makes it relevant for further theoretical study of MAS, the creation of new approaches, the allocation of new families, as well as the development of tools for their description, research and implementation, the deduction of techniques and methods, both justifying the use of existing systems and setting the direction for their improvement and the creation of new systems, having certain properties $[8,9]$.

The basis of a multi-agent approach is the concept of a mobile software agent that is implemented and functions as an independent specialized computer program or an element of artificial intelligence.

Initially, before the advent of relevant information technologies, the "agent" was a person who was delegated some of his authority, both in the performance of specific functions and in decision-making. In the first (non-computer) multiagent systems, agents represented employees of companies on whose behalf and on behalf of whom they interacted with each other when performing a specific task - for example, representatives of the buyer and seller in the trading network or in other types of business. Such systems inherited many of the features of a "bureaucratic" organization, including centralized management, a static structure, and highly specialized agent functionality. Such systems, which replicate the centralized hierarchy, were quickly replaced by distributed systems in which knowledge and resources were distributed among sufficiently "independent" agents, but the general command body of management was maintained, making decisions in critical or conflict situations. A further step in this direction was the paradigm of fully decentralized systems, in which management occurs only through local interactions between agents. At the same time, the narrow functional orientation of the agent to the solution of any one separate part of the general task gradually began to give way to universal integrity (autonomy). Examples of such decentralized organizations include, in part, colonies of insects, for example, bees or ants [10-12].

The essence of multi-agent technology is a fundamentally new method of solving problems. In contrast to the classical method, when some welldefined (deterministic) algorithm is searched, which allows finding the best solution to a problem, in multi-agent technologies, the solution is automatically obtained as a result of the interaction of many independent targeted software modules - so-called software agents.

Intellect is inherent in man - it distinguishes him from a computer acting strictly according to 
the program embedded in it. And this is what allows a person to navigate in a difficult situation, deal with unclear tasks, adapt to changing conditions. Uncertainty is most often present when there is a set of alternatives, and it is impossible to predict which of the options will turn out to be the best after a sufficiently long time.

Every single ant or bee is obviously not intelligent. On the other hand, the colony as a whole shows amazing patterns of behavior, which in many ways can be considered intellectual. Such situations are called a manifestation of an emergent intelligence, or unexpected properties that the system possesses, but no single element within it has. The effect of "intellectual resonance" arising in this case is often called "swarm intelligence". Indeed, the intelligence and physical strength of a single bee are not so great, but a swarm of bees, acting in concert, can defeat a bear and even a person [13].

These scripts are generated and executed by the agents themselves.

Let the state of a weakly formalized process that described by specifying a sample of fuzzy experimental data $\left(X_{r}, y_{r}\right), r=\overline{1, M}$.

Here $X_{r}=\left(x_{r 1}, x_{r 2}, \ldots, x_{r n}\right)$ - the input is a dimensional fuzzy vector, which is defined with its membership functions, and is the corresponding output vector.

It is required to construct fuzzy-correct models of decision-making tasks for estimating and $y_{r}=\left(y_{1}, y_{2}, \ldots, y_{M}\right)$ predicting the state of a poorly formalized process, described in general terms by a set of fuzzy production rules (linguistic statements).

$\bigcup_{p=1}^{k_{i}}\left(\mathrm{I}_{j=1}^{n} w_{i p}^{x_{j}^{i}}=a_{i j}^{p}-\right.$ with weight $) \rightarrow y_{i}^{f}=f\left(b_{i 0}, b_{i 1}, b_{i 2}, \ldots, b_{i n}\right)$

$$
, \quad i=\overline{1, m} \text {. }
$$

Here: - linguistic term, which is estimated variable in the line with the number $\mathrm{p}$ in the rules $\mathrm{i}$ :

- weight coefficient for lines $\mathrm{p}$ in rules $\mathrm{i}$;

- the output of model (1), described by rule $\mathrm{i}$.

It is required to find such values of the unknown coefficients (,) in the process of constructing a fuzzy model (1) that provide a minimum of the residual.

The proposed approach was tested when solving the problem of estimating and forecasting using real data.

The model of the process under study in the described algorithm is represented as a fuzzyneural network (that is, approximated by a neural network with fuzzy parameters).

To solve decision-making problems, models of which are described by fuzzy predicate rules (logical equations) with the corresponding membership functions of fuzzy terms, the following algorithm is proposed $[8,49]$.

1. The values of the object state parameters are recorded:

$$
\text { 2. } \quad X^{*}=\left(x_{1}^{*}, x_{2}^{*}, \ldots, x_{n}^{*}\right) \text {. }
$$

The values of the membership functions are determined at fixed values of the parameters.

3. Using logical equations, the values of the membership functions are calculated for the state vector.

4. Find a solution for which the membership function is defined as:

The essence of learning consists of selecting parameters such the membership functions that minimize the difference between the results of fuzzy-neural approximation and the actual behavior of the object.

The fuzzy-neural network learning algorithm consists of two phases. In the first phase, the model value of the object output () corresponding to the specified network architecture is calculated. In the second phase, the value of the residual is calculated () and the parameters of the membership functions are recalculated.

6. Computational experiment. The task of the computational experiment was to implement and analyze the correctness of the proposed algorithm for solving the problem of making a decision on assessing the state of poorly formalized processes described by a fuzzy-neural network. At the same time, the input data of the 
model were represented by unmanaged parameters.

The fuzzy rules of the knowledge base of each agent follow as:

For the first agent:

$\operatorname{IF}\left[\left(x_{1}=L\right) \quad\right.$ and $\left(x_{2}=L\right) \quad$ and $\left(x_{3}=L\right)$ and $\left.\left(x_{4}=L\right)\right]$

$$
\begin{aligned}
y_{1}= & v_{0}(16,0.98)+v_{1}(47,0.97) x_{1}-v_{2}(669,0.99) x_{2}+v_{3}(374,0.99) x_{3}+v_{4}(28,0.98) x_{4}+ \\
& +v_{5}(35,0.97) x_{1}^{2}-v_{6}(510,0.95) x_{2}^{2}+v_{7}(44,0.97) x_{3}^{2}+v_{8}(5,0.97) x_{4}{ }^{2}+ \\
& +v_{9}(73,0.99) x_{1}^{3}+v_{10}(12,0.97) x_{2}{ }^{3}+v_{11}(5,0.98) x_{3}{ }^{3}-v_{12}(72,0.99) x_{4}{ }^{3}+ \\
& +v_{13}(43,0.96) x_{1}^{4}-v_{14}(5570.99) x_{2}{ }^{4}+v_{15}(73,0.98) x_{3}{ }^{4}+v_{16}(2,0.95) x_{4}{ }^{4}
\end{aligned}
$$

$\operatorname{IF}\left[\left(x_{1}=L\right) \quad\right.$ and $\left(x_{2}=L\right) \quad$ and $\left(x_{3}=L\right)$ and $\left.\left(x_{4}=M\right)\right]$

$$
\begin{aligned}
y_{2}= & v_{0}(16,0.98)+v_{1}(304,0.98) x_{1}-v_{2}(717,0.99) x_{2}+v_{3}(521,0.99) x_{3}-v_{4}(7,0.98) x_{4}+ \\
& -v_{5}(571,0.97) x_{1}^{2}+v_{6}(92,0.98) x_{2}^{2}+v_{7}(73,0.97) x_{3}^{2}+v_{8}(29,0.97) x_{4}{ }^{2}+ \\
& +v_{9}(74,0.98) x_{1}^{3}-v_{10}(433,0.99) x_{2}{ }^{3}+v_{11}(21,0.97) x_{3}{ }^{3}-v_{12}(35,0.99) x_{4}{ }^{3}+ \\
& +v_{13}(83,0.98) x_{1}^{4}+v_{14}(84,0.97) x_{2}{ }^{4}-v_{15}(291,0.99) x_{3}{ }^{4}+v_{16}(2,0.98) x_{4}{ }^{4}
\end{aligned}
$$

$\operatorname{IF}\left[\left(x_{1}=L\right) \quad\right.$ and $\quad\left(x_{2}=L\right) \quad$ and $\quad\left(x_{3}=L\right)$ and $\left.\left(x_{4}=H\right)\right]$

$$
y_{3}=v_{0}(20,0.99)+v_{1}(3925,0.98) x_{1}+v_{2}(345,0.99) x_{2}+v_{3}(128,0.99) x_{3}-v_{4}(47,0.98) x_{4}+
$$

THAT

$$
\begin{aligned}
& +v_{5}(231,0.99) x_{1}^{2}+v_{6}(1923,0.98) x_{2}{ }^{2}-v_{7}(1978,0.95) x_{3}{ }^{2}+v_{8}(588,0.98) x_{4}{ }^{2}+ \\
& -v_{9}(748,0.98) x_{1}{ }^{3}-v_{10}(2483,0.98) x_{2}{ }^{3}+v_{11}(2979,0.95) x_{3}{ }^{3}-v_{12}(552,0.98) x_{4}{ }^{3}+
\end{aligned}
$$$$
+v_{13}(321,0.97) x_{1}^{4}-v_{14}(955,0.99) x_{2}^{4}+v_{15}(543,0.97) x_{3}^{4}+v_{16}(81,0.99) x_{4}^{4}
$$

$\operatorname{IF}\left[\left(x_{1}=L\right)\right.$ and $\left(x_{2}=L\right)$ and $\left(x_{3}=M\right)$ and $\left.\left(x_{4}=L\right)\right]$

$$
\begin{aligned}
y_{4}= & v_{0}(3,0.99)+v_{1}(3925,0.97) x_{1}-v_{2}(3545,0.98) x_{2}+v_{3}(128,0.97) x_{3}-v_{4}(47,0.98) x_{4}+ \\
& -v_{5}(352,0.97) x_{1}^{2}+v_{6}(74,0.98) x_{2}{ }^{2}+v_{7}(398,0.97) x_{3}{ }^{2}-v_{8}(84,0.95) x_{4}{ }^{2}+ \\
& +v_{9}(298,0.98) x_{1}^{3}+v_{10}(375,0.98) x_{2}{ }^{3}+v_{11}(95,0.99) x_{3}^{3}-v_{12}(395,0.97) x_{4}{ }^{3}+ \\
& +v_{13}(289,0.98) x_{1}^{4}+v_{14}(751,0.99) x_{2}{ }^{4}-v_{15}(1357,0.97) x_{3}{ }^{4}+v_{16}(78,0.99) x_{4}{ }^{4}
\end{aligned}
$$

THAT

$\operatorname{IF}\left[\left(x_{1}=L\right)\right.$ and $\left(x_{2}=L\right)$ and $\left(x_{3}=M\right)$ and $\left.\left(x_{4}=M\right)\right]$

$$
\begin{aligned}
y_{5}= & -v_{0}(32,0.98)+v_{1}(135,0.98) x_{1}+v_{2}(354,0.99) x_{2}+v_{3}(99,0.99) x_{3}-\mu_{4}(20,0.98) x_{4}+ \\
& +v_{5}(352,0.97) x_{1}^{2}-v_{6}(95,0.98) x_{2}^{2}-v_{7}(92,0.97) x_{3}^{2}+v_{8}(31,0.97) x_{4}{ }^{2}+ \\
& +v_{9}(43,0.98) x_{1}^{3}-v_{10}(284,0.99) x_{2}^{3}+v_{11}(172,0.97) x_{3}^{3}+v_{12}(49,0.99) x_{4}^{3}+ \\
& +v_{13}(52,0.98) x_{1}^{4}+v_{14}(75,0.97) x_{2}{ }^{4}-v_{15}(332,0.99) x_{3}^{4}+v_{16}(33,0.98) x_{4}^{4}
\end{aligned}
$$

$\operatorname{IF}\left[\left(x_{1}=L\right)\right.$ and $\left(x_{2}=L\right)$ and $\left(x_{3}=M\right)$ and $\left.\left(x_{4}=H\right)\right]$

$$
\begin{aligned}
y_{6}= & v_{0}(12,0.99)-v_{1}(31,0.99) x_{1}-v_{3}(11,0.99) x_{3}-v_{4}(6,0.98) x_{4}+ \\
& +v_{5}(571,0.97) x_{1}^{2}+v_{6}(92,0.98) x_{2}{ }^{2}-v_{7}(673,0.97) x_{3}{ }^{2}+v_{8}(29,0.97) x_{4}{ }^{2}+ \\
& +v_{9}(14,0.95) x_{1}{ }^{3}-v_{10}(23,0.97) x_{2}{ }^{3}+v_{11}(11,0.98) x_{3}{ }^{3}-v_{12}(23,0.97) x_{4}{ }^{3}+ \\
& +v_{13}(22,0.97) x_{1}{ }^{4}+v_{14}(15,0.99) x_{2}{ }^{4}-v_{15}(8,0.97) x_{3}{ }^{4}+v_{16}(13,0.99) x_{4}{ }^{4}
\end{aligned}
$$

THAT

$\operatorname{IF}\left[\left(x_{1}=L\right) \quad\right.$ and $\quad\left(x_{2}=L\right) \quad$ and $\quad\left(x_{3}=H\right) \quad$ and $\left.\quad\left(x_{4}=H\right)\right]$ 


$$
\begin{aligned}
& y_{7}=\mu_{0}(21,0.99)+v_{1}(287,0.98) x_{1}-v_{2}(561,0.99) x_{2}-v_{3}(1,0.99) x_{3}-v_{4}(87,0.98) x_{4}+ \\
& \text { THAT } \\
& -v_{5}(31,0.97) x_{1}^{2}-v_{6}(83,0.98) x_{2}^{2}+v_{7}(54,0.97) x_{3}^{2}+v_{8}(143.97) x_{4}^{2}+ \\
& +v_{9}(32,0.98) x_{1}^{3}+v_{10}(89,0.99) x_{2}^{3}-v_{11}(133,0.97) x_{3}^{3}-v_{12}(42,0.99) x_{4}^{3}+ \\
& +v_{13}(15,0.98) x_{1}^{4}-v_{14}(73,0.97) x_{2}^{4}+v_{15}(51,0.99) x_{3}^{4}+v_{16}(5,0.98) x_{4}^{4} \text {. } \\
& y_{8}=\mu_{0}(8,0.98)+v_{1}(289,0.98) x_{1}+v_{2}(20,0.99) x_{2}-v_{3}(102,0.99) x_{3}-v_{4}(12,0.98) x_{4}+ \\
& -v_{5}(81,0.95) x_{1}^{2}+v_{6}(65,0.97) x_{2}^{2}+v_{7}(29,0.99) x_{3}^{2}+v_{8}(74,0.97) x_{4}^{2}+ \\
& +v_{9}(85,0.99) x_{1}^{3}-v_{10}(39,0.99) x_{2}^{3}+v_{11}(15,0.97) x_{3}^{3}-v_{12}(24,0.98) x_{4}^{3}+ \\
& +v_{13}(83,0.97) x_{1}^{4}+v_{14}(84,0.95) x_{2}^{4}-v_{15}(291,0.94) x_{3}^{4}+v_{16}(2,0.99) x_{4}^{4} \text {. } \\
& y_{9}=v_{0}(55,0.98)+v_{1}(64,0.97) x_{1}-v_{2}(106,0.99) x_{2}-v_{3}(34,0.99) x_{3}+v_{4}(7,0.98) x_{4}+ \\
& +v_{5}(39,0.97) x_{1}^{2}+v_{6}(32,0.98) x_{2}^{2}-v_{7}(171,0.97) x_{3}^{2}+v_{8}(32,0.97) x_{4}^{2}+ \\
& +v_{9}(91,0.98) x_{1}^{3}-v_{10}(56,0.99) x_{2}^{3}+v_{11}(19,0.97) x_{3}^{3}-v_{12}(110.99) x_{4}^{3}+ \\
& -v_{13}(83,0.98) x_{1}^{4}+v_{14}(384,0.97) x_{2}^{4}-v_{15}(291,0.99) x_{3}^{4}+v_{16}(2,0.98) x_{4}^{4} \text {. }
\end{aligned}
$$

For the second agent:

$\operatorname{IF}\left[\left(x_{1}=L\right)\right.$ and $\left(x_{2}=M\right)$ and $\left(x_{3}=L\right)$ and $\left.\left(x_{4}=L\right)\right]$

$$
\begin{aligned}
y_{10}= & v_{0}(27,0.98)-v_{1}(644,0.98) x_{1}-v_{2}(19,0.99) x_{2}-v_{3}(66,0.99) x_{3}+v_{4}(79,0.98) x_{4}+ \\
& -v_{5}(571,0.97) x_{1}^{2}+v_{6}(92,0.98) x_{2}^{2}+v_{7}(73,0.97) x_{3}{ }^{2}+v_{8}(429,0.97) x_{4}{ }^{2}+ \\
& +v_{9}(3,0.98) x_{1}^{3}-v_{10}(8,0.99) x_{2}^{3}+v_{11}(41,0.97) x_{3}^{3}-v_{12}(57,0.99) x_{4}{ }^{3}+ \\
& +v_{13}(2,0.98) x_{1}^{4}+v_{14}(8,0.97) x_{2}{ }^{4}-v_{15}(12,0.99) x_{3}^{4}+v_{16}(7,0.98) x_{4}{ }^{4}
\end{aligned}
$$

THAT

$\operatorname{IF}\left[\left(x_{1}=L\right)\right.$ and $\left(x_{2}=M\right)$ and $\left(x_{3}=L\right)$ and $\left.\left(x_{4}=M\right)\right]$

$$
\begin{aligned}
y_{11}= & v_{0}(8,0.99)-v_{1}(289,0.98) x_{1}+v_{2}(20,0.99) x_{2}-v_{3}(102,0.99) x_{3}-v_{4}(12,0.98) x_{4}+ \\
& -v_{5}(24,0.97) x_{1}{ }^{2}+v_{6}(31,0.98) x_{2}{ }^{2}+v_{7}(27,0.97) x_{3}{ }^{2}+v_{8}(9,0.97) x_{4}{ }^{2}+ \\
& +v_{9}(17,0.98) x_{1}^{3}-v_{10}(74,0.99) x_{2}{ }^{3}+v_{11}(34,0.97) x_{3}{ }^{3}-v_{12}(4,0.99) x_{4}{ }^{3}+ \\
& +v_{13}(23,0.98) x_{1}{ }^{4}+v_{14}(85,0.97) x_{2}{ }^{4}-v_{15}(154,0.99) x_{3}{ }^{4}+v_{16}(83,0.98) x_{4}{ }^{4}
\end{aligned}
$$

THAT

$L$ and $x 2=M$ and $x 3=L$ and $x 4=H$

$$
\begin{aligned}
y_{12}= & v_{0}(55,0.98)-v_{1}(38,0.98) x_{1}-v_{2}(74,0.99) x_{2}-v_{3}(20,0.99) x_{3}-v_{4}(11,0.98) x_{4}+ \\
& -v_{5}(76,0.97) x_{1}^{2}+v_{6}(38,0.98) x_{2}^{2}+v_{7}(21,0.97) x_{3}^{2}+v_{8}(89,0.97) x_{4}^{2}+ \\
& +v_{9}(72,0.98) x_{1}^{3}-v_{10}(292,0.97) x_{2}^{3}+v_{11}(187,0.99) x_{3}^{3}-v_{12}(63,0.97) x_{4}^{3}+ \\
& +v_{13}(25,0.98) x_{1}^{4}+v_{14}(54,0.97) x_{2}^{4}-v_{15}(81,0.98) x_{3}^{4}+v_{16}(17,0.98) x_{4}^{4}
\end{aligned}
$$

$\operatorname{IF}\left[\left(x_{1}=L\right)\right.$ and $\left(x_{2}=M\right)$ and $\left(x_{3}=M\right)$ and $\left.\left(x_{4}=L\right)\right]$ 
$\operatorname{IF}\left[\left(x_{1}=L\right)\right.$ and $\left(x_{2}=M\right)$ and $\left(x_{3}=M\right)$ and $\left.\left(x_{4}=M\right)\right]$

$$
\begin{aligned}
y_{13}= & -v_{0}(15,0.98)-v_{1}(1583,0.99) x_{1}+v_{2}(16,0.99) x_{2}-v_{3}(16,0.99) x_{3}-v_{4}(162,0.98) x_{4}+ \\
& +v_{5}(571,0.97) x_{1}^{2}+v_{6}(92,0.98) x_{2}^{2}-v_{7}(573,0.97) x_{3}^{2}+v_{8}(29,0.97) x_{4}^{2}+ \\
& +v_{9}(44,0.98) x_{1}^{3}-v_{10}(75,0.99) x_{2}^{3}+v_{11}(19,0.97) x_{3}^{3}-v_{12}(64,0.99) x_{4}{ }^{3}+ \\
& +v_{13}(49,0.98) x_{1}^{4}+v_{14}(84,0.97) x_{2}{ }^{4}-v_{15}(125,0.99) x_{3}{ }^{4}+v_{16}(9,0.98) x_{4}{ }^{4}
\end{aligned}
$$

$$
\begin{aligned}
y_{14}= & -v_{0}(25,0.98)-v_{1}(997,0.99) x_{1}+v_{2}(88,0.99) x_{2}+v_{3}(2,0.99) x_{3}+\mu_{4}(94,0.98) x_{4}+ \\
& +v_{5}(79,0.97) x_{1}^{2}+v_{6}(33,0.99) x_{2}^{2}+v_{7}(42,0.97) x_{3}^{2}-v_{8}(265,0.97) x_{4}^{2}+ \\
& +v_{9}(33,0.98) x_{1}^{3}-v_{10}(106,0.99) x_{2}^{3}+v_{11}(45,0.99) x_{3}^{3}-v_{12}(47,0.97) x_{4}^{3}+ \\
& +v_{13}(53,0.97) x_{1}^{4}+v_{14}(47,0.99) x_{2}^{4}-v_{15}(241,0.98) x_{3}^{4}+v_{16}(93,0.98) x_{4}^{4} .
\end{aligned}
$$

$\operatorname{IF}\left[\left(x_{1}=L\right)\right.$ and $\left(x_{2}=M\right)$ and $\left(x_{3}=M\right)$ and $\left.\left(x_{4}=H\right)\right]$

$$
y_{15}=-v_{0}(63,0.98)-v_{1}(7,0.97) x_{1}-v_{2}(12,0.99) x_{2}+v_{3}(179,0.99) x_{3}-v_{4}(16,0.98) x_{4}
$$

THAT

$$
\begin{aligned}
& -v_{5}(45,0.97) x_{1}^{2}+v_{6}(35,0.98) x_{2}^{2}+v_{7}(42,0.97) x_{3}^{2}+v_{8}(74,0.97) x_{4}^{2}+ \\
& +v_{9}(71,0.98) x_{1}^{3}-v_{10}(64,0.99) x_{2}^{3}+v_{11}(85,0.97) x_{3}^{3}-v_{12}(45,0.99) x_{4}{ }^{3}+
\end{aligned}
$$$$
+v_{13}(24,0.98) x_{1}{ }^{4}+v_{14}(71,0.97) x_{2}{ }^{4}-v_{15}(132,0.99) x_{3}{ }^{4}+v_{16}(93,0.98) x_{4}{ }^{4} \text {. }
$$

$\operatorname{IF}\left[\left(x_{1}=L\right) \quad\right.$ and $\quad\left(x_{2}=M\right) \quad$ and $\left(x_{3}=H\right) \quad$ and $\left.\left(x_{4}=M\right)\right]$

$$
\begin{aligned}
y_{16}= & v_{0}(16,0.98)+v_{1}(1240,0.97) x_{1}-v_{2}(3,0.99) x_{2}+v_{3}(7,0.99) x_{3}-v_{4}(120,0.98) x_{4}+ \\
& +v_{5}(71,0.97) x_{1}^{2}-v_{6}(92,0.98) x_{2}^{2}+v_{7}(73,0.97) x_{3}^{2}-v_{8}(59,0.97) x_{4}{ }^{2}+ \\
& +v_{9}(43,0.98) x_{1}^{3}-v_{10}(102,0.99) x_{2}^{3}+v_{11}(10,0.97) x_{3}^{3}-v_{12}(35,0.99) x_{4}{ }^{3}+ \\
& +v_{13}(83,0.98) x_{1}^{4}+v_{14}(84,0.97) x_{2}{ }^{4}-v_{15}(291,0.99) x_{3}{ }^{4}+v_{16}(2,0.98) x_{4}{ }^{4}
\end{aligned}
$$

THAT

$\operatorname{IF}\left[\left(x_{1}=L\right) \quad\right.$ and $\left(x_{2}=M\right)$ and $\left(x_{3}=H\right)$ and $\left.\left(x_{4}=M\right)\right]$

$$
\begin{aligned}
y_{17}= & v_{0}(35,0.98)+v_{1}(317,0.97) x_{1}-v_{2}(19,0.99) x_{2}-v_{3}(4,0.99) x_{3}-v_{4}(28,0.98) x_{4}+ \\
& -v_{5}(33,0.97) x_{1}^{2}+v_{6}(72,0.98) x_{2}^{2}+v_{7}(93,0.97) x_{3}{ }^{2}-v_{8}(28,0.97) x_{4}{ }^{2}+ \\
& +v_{9}(64,20.98) x_{1}^{3}-v_{10}(21,0.99) x_{2}^{3}+v_{11}(32,0.97) x_{3}{ }^{3}-v_{12}(55,0.99) x_{4}{ }^{3}+ \\
& +v_{13}(9,0.98) x_{1}^{4}-v_{14}(34,0.97) x_{2}{ }^{4}-v_{15}(36,0.99) x_{3}{ }^{4}+v_{16}(81,0.98) x_{4}{ }^{4} .
\end{aligned}
$$

$\operatorname{IF}\left[\left(x_{1}=L\right)\right.$ and $\left(x_{2}=M\right)$ and $\left(x_{3}=H\right)$ and $\left.\left(x_{4}=H\right)\right]$

$$
\begin{aligned}
y_{18}= & v_{0}(85,0.98)-v_{1}(223,0.97) x_{1}-v_{2}(55,0.99) x_{2}-v_{3}(36,0.99) x_{3}+v_{4}(26,0.98) x_{4}+ \\
& +v_{5}(32,0.97) x_{1}{ }^{2}-v_{6}(72,0.98) x_{2}{ }^{2}-v_{7}(45,0.97) x_{3}{ }^{2}+v_{8}(89,0.97) x_{4}{ }^{2}+ \\
& +v_{9}(82,0.98) x_{1}^{3}-v_{10}(171,0.97) x_{2}{ }^{3}+v_{11}(81,0.97) x_{3}{ }^{3}-v_{12}(11,0.399) x_{4}{ }^{3}+ \\
& +v_{13}(21,0.97) x_{1}{ }^{4}+v_{14}(33,0.99) x_{2}{ }^{4}-v_{15}(92,0.99) x_{3}{ }^{4}+v_{16}(45,0.98) x_{4}{ }^{4} .
\end{aligned}
$$

For the third agent:

$\operatorname{IF}\left[\left(x_{1}=L\right) \quad\right.$ and $\left(x_{2}=M\right) \quad$ and $\left(x_{3}=H\right) \quad$ and $\left.\left(x_{4}=M\right)\right]$ 


$$
\begin{aligned}
y_{19}= & -v_{0}(5,0.99)+v_{1}(655,0.97) x_{1}+v_{2}(25,0.99) x_{2}-v_{3}(700,0.99) x_{3}-v_{4}(53,0.98) x_{4}+ \\
& -v_{5}(7,0.97) x_{1}^{2}+v_{6}(12,0.98) x_{2}{ }^{2}+v_{7}(33,0.97) x_{3}^{2}-v_{8}(349,0.97) x_{4}^{2}+ \\
& +v_{9}(54,0.98) x_{1}^{3}-v_{10}(79,0.99) x_{2}^{3}+v_{11}(34,0.97) x_{3}^{3}-v_{12}(12,0.99) x_{4}{ }^{3}+ \\
& +v_{13}(24,0.98) x_{1}^{4}+v_{14}(23,0.97) x_{2}{ }^{4}-v_{15}(52,0.99) x_{3}{ }^{4}+v_{16}(11,0.98) x_{4}{ }^{4} .
\end{aligned}
$$

$\operatorname{IF}\left[\left(x_{1}=L\right)\right.$ and $\left(x_{2}=H\right)$ and $\left(x_{3}=L\right)$ and $\left.\left(x_{4}=M\right)\right]$

$$
\begin{aligned}
y_{20}= & -v_{0}(104,0.98)+v_{1}(20,0.98) x_{1}-v_{2}(227,0.99) x_{2}+v_{3}(37,0.99) x_{3}-v_{4}(53,0.98) x_{4}+ \\
& -v_{5}(271,0.97) x_{1}^{2}+v_{6}(54,0.98) x_{2}{ }^{2}-v_{7}(91,0.97) x_{3}{ }^{2}+v_{8}(182,0.97) x_{4}{ }^{2}+ \\
& +v_{9}(133,0.98) x_{1}^{3}-v_{10}(31,0.99) x_{2}{ }^{3}+v_{11}(53,0.97) x_{3}{ }^{3}-v_{12}(97,0.99) x_{4}{ }^{3}+ \\
& +v_{13}(24,0.98) x_{1}{ }^{4}-v_{14}(75,0.97) x_{2}{ }^{4}+v_{15}(61,0.99) x_{3}{ }^{4}-v_{16}(23,0.98) x_{4}{ }^{4} .
\end{aligned}
$$

$\operatorname{IF}\left[\left(x_{1}=M\right)\right.$ and $\left(x_{2}=M\right)$ and $\left(x_{3}=M\right)$ and $\left.\left(x_{4}=H\right)\right]$

$$
y_{21}=-v_{0}(240,0.98)-v_{1}(236,0.98) x_{1}-v_{2}(350,0.99) x_{2}+v_{3}(146,0.99) x_{3}+v_{4}(47,0.98) x_{4}+
$$

THAT

$$
\begin{aligned}
& -v_{5}(41,0.97) x_{1}^{2}+v_{6}(90,0.98) x_{2}{ }^{2}+v_{7}(83,0.97) x_{3}{ }^{2}-v_{8}(129,0.97) x_{4}{ }^{2}+ \\
& +v_{9}(21,0.98) x_{1}{ }^{3}-v_{10}(32,0.99) x_{2}{ }^{3}+v_{11}(47,0.97) x_{3}{ }^{3}-v_{12}(29,0.99) x_{4}{ }^{3}+ \\
& +v_{13}(83,0.98) x_{1}{ }^{4}+v_{14}(84,0.97) x_{2}{ }^{4}-v_{15}(291,0.99) x_{3}{ }^{4}+v_{16}(2,0.98) x_{4}{ }^{4}
\end{aligned}
$$

$\operatorname{IF}\left[\left(x_{1}=M\right)\right.$ and $\left(x_{2}=M\right)$ and $\left(x_{3}=H\right)$ and $\left.\left(x_{4}=M\right)\right]$

$$
\begin{aligned}
y_{22}= & v_{0}(226,0.98)-v_{1}(132,0.98) x_{1}-v_{2}(159,0.99) x_{2}-v_{3}(59,0.99) x_{3}+v_{4}(27,0.98) x_{4}+ \\
& -v_{5}(421,0.97) x_{1}^{2}+v_{6}(57,0.98) x_{2}{ }^{2}+v_{7}(212,0.97) x_{3}{ }^{2}+v_{8}(221,0.97) x_{4}{ }^{2}+ \\
& +v_{9}(81,0.98) x_{1}^{3}-v_{10}(121,0.99) x_{2}{ }^{3}+v_{11}(92,0.97) x_{3}{ }^{3}-v_{12}(55,0.99) x_{4}{ }^{3}+ \\
& +v_{13}(83,0.98) x_{1}{ }^{4}+v_{14}(84,0.97) x_{2}{ }^{4}-v_{15}(291,0.99) x_{3}{ }^{4}+v_{16}(2,0.98) x_{4}{ }^{4} . \\
y_{23} & =v_{0}(43,0.98)-v_{1}(35,0.99) x_{1}-v_{2}(7,0.99) x_{2}+v_{3}(42,0.99) x_{3}-v_{4}(6,0.99) x_{4}+ \\
& +v_{5}(81,0.97) x_{1}^{2}+v_{6}(49,0.98) x_{2}{ }^{2}-v_{7}(273,0.97) x_{3}{ }^{2}+v_{8}(129,0.97) x_{4}{ }^{2}+ \\
& +v_{9}(221,0.98) x_{1}^{3}-v_{10}(92,0.99) x_{2}{ }^{3}+v_{11}(31,0.97) x_{3}{ }^{3}-v_{12}(235,0.99) x_{4}{ }^{3}+ \\
& +v_{13}(31,0.98) x_{1}{ }^{4}+v_{14}(23,0.97) x_{2}{ }^{4}-v_{15}(67,0.99) x_{3}{ }^{4}+v_{16}(33,0.98) x_{4}{ }^{4} .
\end{aligned}
$$

$\operatorname{IF}\left[\left(x_{1}=H\right)\right.$ and $\left(x_{2}=H\right)$ and $\left(x_{3}=M\right)$ and $\left.\left(x_{4}=H\right)\right]$

$$
\begin{aligned}
y_{24}= & v_{0}(11,0.99)-v_{1}(1,0.98) x_{1}+v_{2}(5,0.99) x_{2}+v_{3}(12,0.98) x_{3}+v_{4}(1,0.99) x_{4}+ \\
& +v_{5}(12,0.97) x_{1}^{2}+v_{6}(21,0.97) x_{2}{ }^{2}+v_{7}(3,0.97) x_{3}^{2}-v_{8}(42,0.97) x_{4}{ }^{2}+ \\
& +v_{9}(14,0.99) x_{1}^{3}-v_{10}(7,0.99) x_{2}{ }^{3}+v_{11}(31,0.97) x_{3}{ }^{3}-v_{12}(42,0.98) x_{4}{ }^{3}+ \\
& +v_{13}(23,0.98) x_{1}^{4}+v_{14}(12,0.97) x_{2}{ }^{4}-v_{15}(31,0.99) x_{3}{ }^{4}-v_{16}(4,0.98) x_{4}{ }^{4} .
\end{aligned}
$$

$\operatorname{IF}\left[\left(x_{1}=H\right)\right.$ and $\left(x_{2}=H\right)$ and $\left(x_{3}=H\right)$ and $\left.\left(x_{4}=L\right)\right]$

THAT

$$
\begin{aligned}
y_{25} & =v_{0}(15,0.98)-v_{1}(13,0.99) x_{1}+v_{2}(4,0.99) x_{2}+v_{3}(15,0.99) x_{3}+v_{4}(3,0.99) x_{4}+ \\
+ & v_{5}(12,0.97) x_{1}{ }^{2}+v_{6}(23,0.98) x_{2}{ }^{2}-v_{7}(31,0.97) x_{3}{ }^{2}+v_{8}(4,0.97) x_{4}{ }^{2}+ \\
& +v_{9}(21,0.98) x_{1}^{3}-v_{10}(54,0.99) x_{2}^{3}+v_{11}(42,0.97) x_{3}^{3}-v_{12}(9,0.99) x_{4}{ }^{3}+ \\
& +v_{13}(23,0.98) x_{1}^{4}+v_{14}(18,0.97) x_{2}{ }^{4}-v_{15}(32,0.99) x_{3}{ }^{4}-v_{16}(5,0.98) x_{4}{ }^{4} .
\end{aligned}
$$

$\operatorname{IF}\left[\left(x_{1}=H\right)\right.$ and $\left(x_{2}=H\right)$ and $\left(x_{3}=H\right)$ and $\left.\left(x_{4}=M\right)\right]$ 


$$
\begin{aligned}
& y_{26}=v_{0}(97,0.99)-v_{1}(34,0.98) x_{1}-v_{2}(84,0.99) x_{2}+v_{3}(43,0.99) x_{3}-v_{4}(5,0.99) x_{4}+ \\
& +v_{5}(81,0.97) x_{1}^{2}-v_{6}(31,0.97) x_{2}^{2}-v_{7}(29,0.97) x_{3}^{2}-v_{8}(18,0.97) x_{4}^{2}+ \\
& \text { THAT } \\
& +v_{9}(11,0.98) x_{1}^{3}-v_{10}(32,0.97) x_{2}^{3}+v_{11}(22,0.99) x_{3}^{3}-v_{12}(4,0.98) x_{4}^{3}+ \\
& +v_{13}(35,0.98) x_{1}^{4}+v_{14}(24,0.98) x_{2}^{4}-v_{15}(61,0.97) x_{3}^{4}+v_{16}(7,0.99) x_{4}^{4} \text {. } \\
& \operatorname{IF}\left[\left(x_{1}=H\right) \text { and }\left(x_{2}=H\right) \text { and }\left(x_{3}=H\right) \text { and }\left(x_{4}=H\right)\right] \\
& y_{27}=v_{0}(52,0.98)-v_{1}(27,0.98) x_{1}-v_{2}(2,0.99) x_{2}-v_{3}(1,0.99) x_{3}-v_{4}(1,0.99) x_{4}+ \\
& -v_{5}(41,0.97) x_{1}^{2}+v_{6}(14,0.98) x_{2}^{2}-v_{7}(64,0.97) x_{3}^{2}+v_{8}(12,0.97) x_{4}^{2}+ \\
& \text { THAT } \\
& +v_{9}(23,0.98) x_{1}^{3}-v_{10}(51,0.99) x_{2}^{3}+v_{11}(32,0.97) x_{3}^{3}-v_{12}(7,0.99) x_{4}^{3}+ \\
& +v_{13}(39,0.98) x_{1}{ }^{4}-v_{14}(41,0.97) x_{2}{ }^{4}+v_{15}(31,0.99) x_{3}{ }^{4}-v_{16}(28,0.98) x_{4}{ }^{4} \text {. }
\end{aligned}
$$

The results obtained showed the high efficiency of the proposed algorithm for solving the problem of decision making in forecasting, classifying and evaluating poorly formalized processes described by fuzzy models.

\section{CONCLUSION}

An analysis of the research has shown that the alternative concept of multi-agent distributed intelligent systems with interaction and competition among agents differs from the classical approach as follows: each intelligent agent operates completely autonomously; each intelligent agent offers a solution to a common problem, each agent has full access to all available information; A common solution to a problem is defined as the proposal of one of the parallel-functioning agents on the basis of competition, while the cooperation of agents forms the necessary behavior of the entire system; actions that related to cooperation and competition in the system are carried out simultaneously (not consistently). The essence of multi-agent modeling can generally be divided into two classes: ordinary, traditional objects of the environment, which can be considered passive, since they are waiting for a message before performing the operation. After the objects are initiated, they perform their functions and "fall asleep" until they receive the next task. The use of fuzzy inference in the reasoning of the IA allows you to avoid the cumbersome rules, compared with the production approach, to increase the speed of calculating the result and expand its spectrum by increasing the power of the term-sets, which brings the reasoning of the AI to the human ones. The use of the Mamdani method is explained by the simplicity of the software implementation and the naturalness of the results obtained.

\section{REFERENCES}

1. R.A. Aliev, R.R. Aliev The theory of intelligent systems and its application. - Baku, Chashyogly Publishing House, 2001. -720 p.

2. Rear. L.A. The concept of a linguistic variable
and its application to making approximate

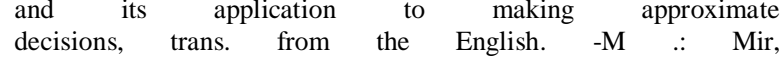
1976. $-165 \mathrm{c}$.

3. YermolievYu.M. Methods of stochastic programming. M $\quad \therefore \quad$ Science, 1976. -240 p.

4. Mikhalevich, VS, Knopov, PS, Golodnikov, AN Mathematical models and risk assessment methods for environmentally hazardous industries // Cybernetics and systems analysis. 1994. №2. WITH. 121-138.

5. Nedosekin A.O. Fuzzy-multiple risk analysis of stock investments. $\mathrm{SPb}$ : Sesame. 2002. $-181 \mathrm{p}$.

6. Zadeh L. The Information principle // In Proc. the 15th IEEE International Conference on Information Reuse and Integration, San Francisco, CA, USA; August 13-15, 2014.

7. Bordini Rafael H. Multi-Agent Programming: Languages, Platforms and Applications / Rafael H. Bordini, Mehdi Dastani, Jurgen Dix, Amal El FallahSeghrouchni. - Springer, 2005. - 296 p

8. Hong Huang, Zhengxiong Zhang. Research Status and Prospect of Vehicle Routing Problem // Logistics technology, 2010.10 (227): pp.21-24.

9. Nourossana S, Erfani H. Bee colony system: safety and discretion optimization // International Journal on Artificial Intelligence Tools, 2012,21 (1): pp.6-16.

10. HuiLan, Qinfei He. Optimization of the Road Accessory // Journal of Dalian Maritime $\begin{array}{lll}\text { University, } 2015.41 & (4): \quad \text { pp.67-74. }\end{array}$

11. Lin Hong. Architectural Design of Multi-Agent Systems // Premier Reference Series / Hong

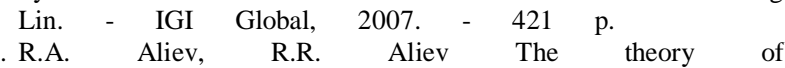

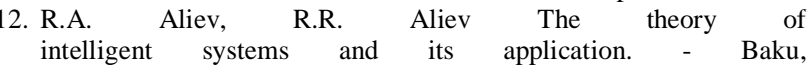
Chashyogly Publishing House, 2001. -720 p.
Rear. L.A. The concept of a linguistic variable

13. Rear. L.A. The concept of a linguistic variable
and its application to making approximate decisions, trans. from the English. -M $\therefore$ Mir,
1976. -165c.

14. YermolievYu.M. Methods of stochastic programming. M : Science, 1976. -240 p.

15. Mikhalevich, VS, Knopov, PS, Golodnikov, AN Mathematical models and risk assessment methods for environmentally hazardous industries // Cybernetics and systems analysis. 1994. №2. WITH. 121-138.

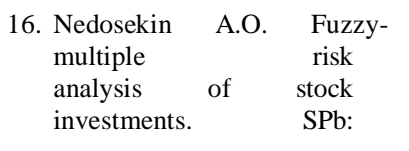

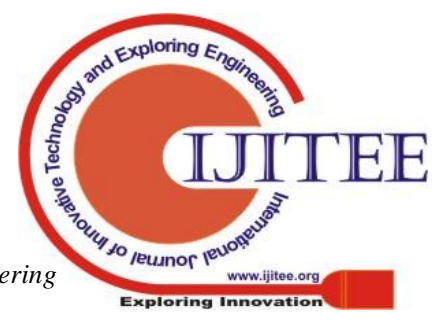


Sesame. 2002. -181 p.

17. Zadeh L. The Information principle // In Proc. the 15th IEEE International Conference on Information Reuse and Integration, San Francisco, CA, USA; August 13-15, 2014.

18. Bordini Rafael H. Multi-Agent Programming: Languages, Platforms and Applications / Rafael H. Bordini, Mehdi Dastani, Jurgen Dix, Amal El FallahSeghrouchni. - Springer, 2005. - 296 p.

19. Hong Huang, Zhengxiong Zhang. Research Status and Prospect of Vehicle Routing Problem // Logistics technology, $2010.10 \quad$ (227): pp.21-24.

20. Nourossana S, Erfani H. Bee colony system: safety and discretion optimization // International Journal on Artificial Intelligence Tools, 2012,21 (1): pp.6-16.

21. intelligent systems and its application. - Baku, Chashyogly Publishing House, 2001. -720 p.

22. Rear. L.A. The concept of a linguistic variable and its application to making approximate decisions, trans. from the English. -M $\therefore$ Mir, 1976. $-165 \mathrm{c}$.

23. YermolievYu.M. Methods of stochastic programming. M $\quad \therefore \quad$ Science, $1976 . \quad-240 \quad \mathrm{p}$

24. Mikhalevich, VS, Knopov, PS, Golodnikov, AN Mathematical models and risk assessment methods for environmentally hazardous industries // Cybernetics and systems analysis. 1994. №2. WITH. 121-138.

25. Nedosekin A.O. Fuzzy-multiple risk analysis of stock investments. SPb: Sesame. 2002. -181 p.

26. intelligent systems and its application. - Baku, Chashyogly Publishing House, 2001. -720 p.

27. Rear. L.A. The concept of a linguistic variable and its application to making approximate decisions, trans. from the English. -M.$:$ Mir, 1976. -165c.

28. YermolievYu.M. Methods of stochastic programming. M $\quad \therefore \quad$ Science, $1976 . \quad-240$ p

29. Mikhalevich, VS, Knopov, PS, Golodnikov, AN Mathematical models and risk assessment methods for environmentally hazardous industries // Cybernetics and systems analysis. 1994. -№2. WITH. 121-138.

30. Nedosekin A.O. Fuzzy-multiple risk analysis of stock investments. SPb: Sesame. 2002. -181 p.

31. Zadeh L. The Information principle // In Proc. the 15th IEEE International Conference on Information Reuse and Integration, San Francisco, CA, USA; August 13-15, 2014.

32. Bordini Rafael H. Multi-Agent Programming: Languages, Platforms and Applications / Rafael H. Bordini, Mehdi Dastani, Jurgen Dix, Amal El FallahSeghrouchni. - Springer, 2005. - 296 p

33. Hong Huang, Zhengxiong Zhang. Research Status and Prospect of Vehicle Routing Problem // Logistics technology, 2010.10 (227): pp.21-24.

34. Nourossana S, Erfani H. Bee colony system: safety and discretion optimization // International Journal on Artificial Intelligence Tools, 2012,21 (1): pp.6-16.

35. HuiLan, Qinfei He. Optimization of the Road Accessory // Journal of Dalian Maritime University, $2015.41 \quad$ (4): $\quad$ pp.67-74

36. Lin Hong. Architectural Design of Multi-Agent Systems // Premier Reference Series / Hong Lin. - IGI Global, 2007. - 421 p.

37. R.A. Aliev, R.R. Aliev The theory of intelligent systems and its application. - Baku, Chashyogly Publishing House, 2001. -720 p.

38. Rear. L.A. The concept of a linguistic variable
and its application to making approximate

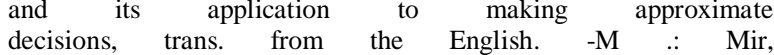
1976. -165c.

39. YermolievYu.M. Methods of stochastic programming. M .: Science, 1976. -240 p.

40. Mikhalevich, VS, Knopov, PS, Golodnikov, AN Mathematical models and risk assessment methods for environmentally hazardous industries //
Cybernetics and

WITH. 121-138.

41. Nedosekin A.O. Fuzzy-multiple risk analysis of stock investments. $\mathrm{SPb}$ : Sesame. 2002. -181 p.

42. Zadeh L. The Information principle // In Proc. the 15th IEEE International Conference on Information Reuse and Integration, San Francisco, CA, USA; August 13-15, 2014.

43. Bordini Rafael H. Multi-Agent Programming: Languages, Platforms and Applications / Rafael H. Bordini, Mehdi Dastani, Jurgen Dix, Amal El FallahSeghrouchni. - Springer, 2005. - 296 p.

44. Hong Huang, Zhengxiong Zhang. Research Status and Prospect of Vehicle Routing Problem // Logistics technology, 2010.10 (227): pp.21-24.

45. Nourossana S, Erfani H. Bee colony system: safety and discretion optimization // International Journal on Artificial Intelligence Tools, 2012,21 (1): pp.6-16.

46. Huilan, Qinfei He. Optimization of the Road Accessory // Journal of Dalian Maritime University, $2015.41 \quad$ (4): $\quad$ pp.67-74.

47. Lin Hong. Architectural Design of Multi-Agent Systems // Premier Reference Series / Hong Lin. - IGI Global, 2007. - 421 p.

48. Shamma Jeff. Cooperative Control of Distributed Multi-Agent Systems. - John Wiley \& Sons, 2008. - 437 p.

49. Alain Fougires. A Modeling Approach Based On Fuzzy Agents // International Journal Of Computer Science Issues, November, 2012.

50. Xiaojun Bi. Improved Artificial Bee Colony Algorithm // Journal of Harbin Engineering University, $2012.33 \quad$ (1): pp.117-123

51. Shamma Jeff. Cooperative Control of Distributed Multi-Agent Systems. - John Wiley \& Sons, 2008. - 437 p.

52. Alain Fougires. A Modeling Approach Based On Fuzzy Agents // International Journal O Computer Science Issues, November, 2012.

53. Xiaojun Bi. Improved Artificial Bee Colony Algorithm // Journal of Harbin Engineering University, $2012.33 \quad$ (1): pp.117-123

55. Zadeh L. The Information principle // In Proc. the 15th IEEE International Conference on Information Reuse and Integration, San Francisco, CA, USA; August 13-15, 2014.

56. Bordini Rafael H. Multi-Agent Programming: Languages, Platforms and Applications / Rafael H. Bordini, Mehdi Dastani, Jurgen Dix, Amal El FallahSeghrouchni. - Springer, 2005. - 296 p.

57. Hong Huang, Zhengxiong Zhang. Research Status and Prospect of Vehicle Routing Problem // Logistics technology, $2010.10 \quad$ (227): pp.21-24.

58. Nourossana S, Erfani H. Bee colony system: safety and discretion optimization // International Journal on Artificial Intelligence Tools, 2012,21 (1): pp.6-16.

59. HuiLan, Qinfei He. Optimization of the Road Accessory // Journal of Dalian Maritime University, $2015.41 \quad$ (4): pp.67-74.

60. Lin Hong. Architectural Design of Multi-Agent Systems // Premier Reference Series / Hong Lin. - IGI Global, 2007. - 421 p.

61. R.A. Aliev, R.R. Aliev The theory of intelligent systems and its application. - Baku, Chashyogly Publishing House, 2001. -720 p.

62. Rear. L.A. The concept of a linguistic variable and its application to making approximate decisions, trans. from the English. $-\mathrm{M}$. 1976. -165c

63. YermolievYu.M. Methods of stochastic programming. M $\quad \therefore \quad$ Science, 1976. -240 p.

64. Mikhalevich, VS, Knopov, PS, Golodnikov, AN Mathematical models and risk assessment methods for environmentally hazardous industries // 
$\begin{array}{lllll}\text { Cybernetics and systems } & \text { analysis. } 1994 . & \text { №2. }\end{array}$

65. Nedosekin A.O. Fuzzy-multiple risk analysis of stock investments. $\mathrm{SPb}$ : Sesame. 2002. -181 p.

66. Zadeh L. The Information principle // In Proc. the 15th IEEE International Conference on Information Reuse and Integration, San Francisco, CA, USA; August 13-15, 2014.

67. Bordini Rafael H. Multi-Agent Programming: Languages, Platforms and Applications / Rafael H. Bordini, Mehdi Dastani, Jurgen Dix, Amal El FallahSeghrouchni. - Springer, 2005. - 296 p

68. Hong Huang, Zhengxiong Zhang. Research Status and Prospect of Vehicle Routing Problem // Logistics technology, $2010.10 \quad$ (227): pp.21-24.

69. Nourossana S, Erfani H. Bee colony system: safety and discretion optimization // International Journal on Artificial Intelligence Tools, 2012,21 (1): pp.6-16.

70. HuiLan, Qinfei He. Optimization of the Road Accessory // Journal of Dalian Maritime University, $2015.41 \quad$ (4): pp.67-74.

71. Lin Hong. Architectural Design of Multi-Agent Systems // Premier Reference Series / Hong Lin. - IGI Global, 2007. - 421 p.

72. Shamma Jeff. Cooperative Control of Distributed Multi-Agent Systems. - John Wiley \& Sons, 2008. - 437 p.

73. Alain Fougires. A Modeling Approach Based On Fuzzy Agents // International Journal Of Computer Science Issues, November, 2012.

74. Xiaojun Bi. Improved Artificial Bee Colony Algorithm // Journal of Harbin Engineering University, $2012.33 \quad$ (1): pp.117-123

75. Shamma Jeff. Cooperative Control of Distributed Multi-Agent Systems. - John Wiley \& Sons, 2008. - 437 p.

76. Alain Fougires. A Modeling Approach Based On Fuzzy Agents // International Journal Of Computer Science Issues, November, 2012.

77. Xiaojun Bi. Improved Artificial Bee Colony Algorithm // Journal of Harbin Engineering University, $2012.33 \quad$ (1): pp.117-123

78.

79. HuiLan, Qinfei He. Optimization of the Road Accessory // Journal of Dalian Maritime University, $2015.41 \quad$ (4): $\quad$ pp.67-74.

80. Lin Hong. Architectural Design of Multi-Agent Systems // Premier Reference Series / Hong Lin. - IGI Global, 2007. - 421 p.

81. Shamma Jeff. Cooperative Control of Distributed Multi-Agent Systems. - John Wiley \& Sons, 2008. - 437 p.

82. Alain Fougires. A Modeling Approach Based On Fuzzy Agents // International Journal Of Computer Science Issues, November, 2012.

83. Xiaojun Bi. Improved Artificial Bee Colony Algorithm // Journal of Harbin Engineering University, $2012.33 \quad$ (1): pp.117-123

84. Shamma Jeff. Cooperative Control of Distributed Multi-Agent Systems. - John Wiley \& Sons, 2008. - 437 p.

85. Alain Fougires. A Modeling Approach Based On Fuzzy Agents // International Journal Of Computer Science Issues, November, 2012. 